Supporting Information

\title{
Multifunctional Antimicrobial Polypeptide-Selenium Nanoparticles Combat Drug-Resistant Bacteria
}

Tao Huang ${ }^{1,2}$, James A. Holden ${ }^{2}$, Eric C. Reynolds ${ }^{2}$, Daniel E. Heath ${ }^{1}$, Neil M. O’Brien-Simpson ${ }^{2}$, and Andrea J. O’Connor ${ }^{1, *}$

\footnotetext{
${ }^{1}$ Department of Biomedical Engineering, University of Melbourne, Parkville, VIC 3010, Australia.

${ }^{2}$ Melbourne Dental School and The Bio21 Institute of Molecular Science and Biotechnology, The University of Melbourne, Parkville, VIC 3010, Australia.

* corresponding author; a.oconnor@unimelb.edu.au
} 


\section{Materials}

Selenium dioxide $\left(\mathrm{SeO}_{2}\right)$ and polyvinyl alcohol (PVA, MW 9000-10000, 80\% hydrolysed) were purchased from Sigma Aldrich (Castle Hill, NSW, Australia). Sodium thiosulphate $\left(\mathrm{Na}_{2} \mathrm{~S}_{2} \mathrm{O}_{3}\right)$ was obtained from Science Supply Australia (Mitcham, VIC, Australia). $\varepsilon$-poly-Llysine with average molecular weight of 3500-4500 Da was purchased from Carbosynth (Berkshire, UK). PBS tablets were purchased from Gibco (UK). All water used was purified by a Milli-Q water purification system (Merck Millipore, Massachusetts, USA) to a resistivity of $\geq 18.2 \mathrm{M} \Omega \cdot \mathrm{cm}$.

\section{Methods}

\section{Bacterial zeta potential measurements}

The bacterial strains methicillin-sensitive S. aureus, E. faecalis, E. coli, A. baumannii and $K$. pneumoniae were inoculated from agar plates into MHB for overnight culture. The bacteria suspensions obtained were centrifuged with at $16000 \mathrm{~g}$ for $20 \mathrm{~min}$. at $37{ }^{\circ} \mathrm{C}$, then washed with sterilized water three times and resuspended into PBS solution. $10 \mu \mathrm{L}$ of the resuspended bacteria suspension was diluted to $1 \mathrm{~mL}$ in PBS solution for zeta potential measurements using a Zetasizer (Malvern, ATA Scientific). Each sample was measured three times.

\section{Cytotoxicity tests on human dermal fibroblasts}

Human dermal fibroblasts (HDFs) were cultured in DMEM with $10 \%$ FBS, $100 \mathrm{U} / \mathrm{mL}$ penicillin and $100 \mu \mathrm{g} / \mathrm{mL}$ streptomycin at $37{ }^{\circ} \mathrm{C}$ in a humidified atmosphere of $5 \% \mathrm{CO}_{2}$. Two methods were adopted to test the cytotoxicity of Se NPs, Se NP- $\varepsilon$-PL and $\varepsilon$-PL, namely the Cell Counting Kit-8 (CCK-8) assay and Lactate Dehydrogenase (LDH) assay.

For the CCK-8 tests, filter sterilized Se NPs, Se NP- $\varepsilon$-PL and pure $\varepsilon$-PL in PBS solutions were diluted in PBS to concentrations of 20, 50, 100, 200, 500 and $1000 \mu \mathrm{g} / \mathrm{mL}$ and $5 \%(\mathrm{v} / \mathrm{v})$ of these diluted solutions was added to DMEM to give final concentrations of Se NPs of 1, 2.5, $5,10,25$ and $50 \mu \mathrm{g} / \mathrm{mL}$. The control groups involved DMEM as the negative control and DMEM with $10 \%$ dimethyl sulfoxide (DMSO) as the positive control, according to the ISO 10993-5 standard. ${ }^{1}$ Cells were initially incubated in 96-well plates at a density of approximately $5 \times 10^{3}$ cells per $100 \mu \mathrm{L}$ DMEM per well for $24 \mathrm{~h}$ to allow attachment. The DMEM was then replaced with $100 \mu \mathrm{L}$ DMEM with Se NPs, Se NP- $\varepsilon$-PL or pure $\varepsilon$-PL. After $24 \mathrm{~h}$ incubation, the medium was removed, and the cultures were washed once with PBS. $120 \mu \mathrm{L}$ of DMEM with 10\% CCK-8 solution was then added to each well and incubation was continued for $3 \mathrm{~h}$. $100 \mu \mathrm{L}$ medium from each well was transferred to new 96-well plates and the absorbance of each well at $450 \mathrm{~nm}$ was tested with a microplate reader (M200 PRO, Tecan). The cell viability $(X)$ of each experimental group was calculated based on 5 samples according to Equation (2) where $\mathrm{OD}_{1}$ represents the mean absorbance of each of the experimental groups and the positive control group and $\mathrm{OD}_{2}$ represents the mean absorbance of the negative control group.

$$
X=\frac{O D_{1}}{O D_{2}} \times 100 \%
$$

LDH is released from cells into the medium when cell lysis occurs. The amount of LDH released to the medium was measured using the Cyto Tox $96{ }^{\circledR}$ nonradioactive assay (Promega, Madison, WI, USA) following the manufacturer's instructions. The HDFs were cultured in a 
96-well microplate for $24 \mathrm{~h}$, then the medium was replaced with DMEM with Se NPs, Se NP$\varepsilon$-PL or pure $\varepsilon$-PL. Pure DMEM was used as the negative control. The microplate was incubated at $37^{\circ} \mathrm{C}$ for a further $6 \mathrm{~h}$. For the maximum LDH release control, HDFs were lysed using $1 \mathrm{X}$ lysis solution for 45 minutes before adding Cyto Tox $96^{\circledR}$ reagent. After that, Cyto Tox $96^{\circledR}$ reagent was added to the wells and incubated for $30 \mathrm{~min}$. Then the reaction was halted by adding the stop solution. A microplate reader (M200 PRO, Tecan) was used to measure the absorbance at $490 \mathrm{~nm}$. The cytotoxicity (Y) was calculated according to the Equation (3) where $\mathrm{OD}_{3}$ represents the mean absorbance of each of the experimental groups and $\mathrm{OD}_{4}$ represents the mean absorbance of the maximum LDH release control group. All absorbance values were used after subtraction of the culture medium background values.

$$
Y=\frac{O D_{3}}{O D_{4}} \times 100 \%
$$




\section{Supporting figures}
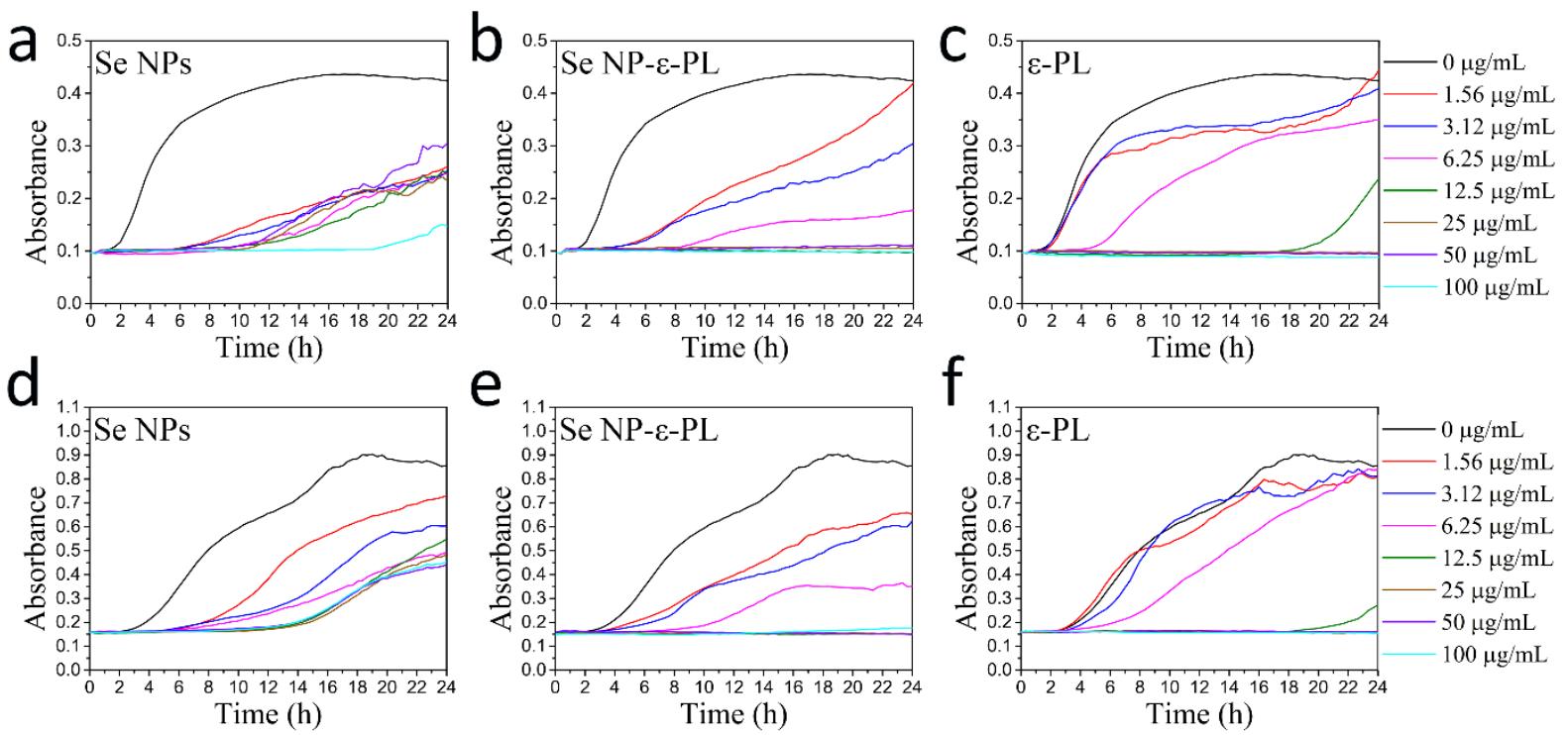

e
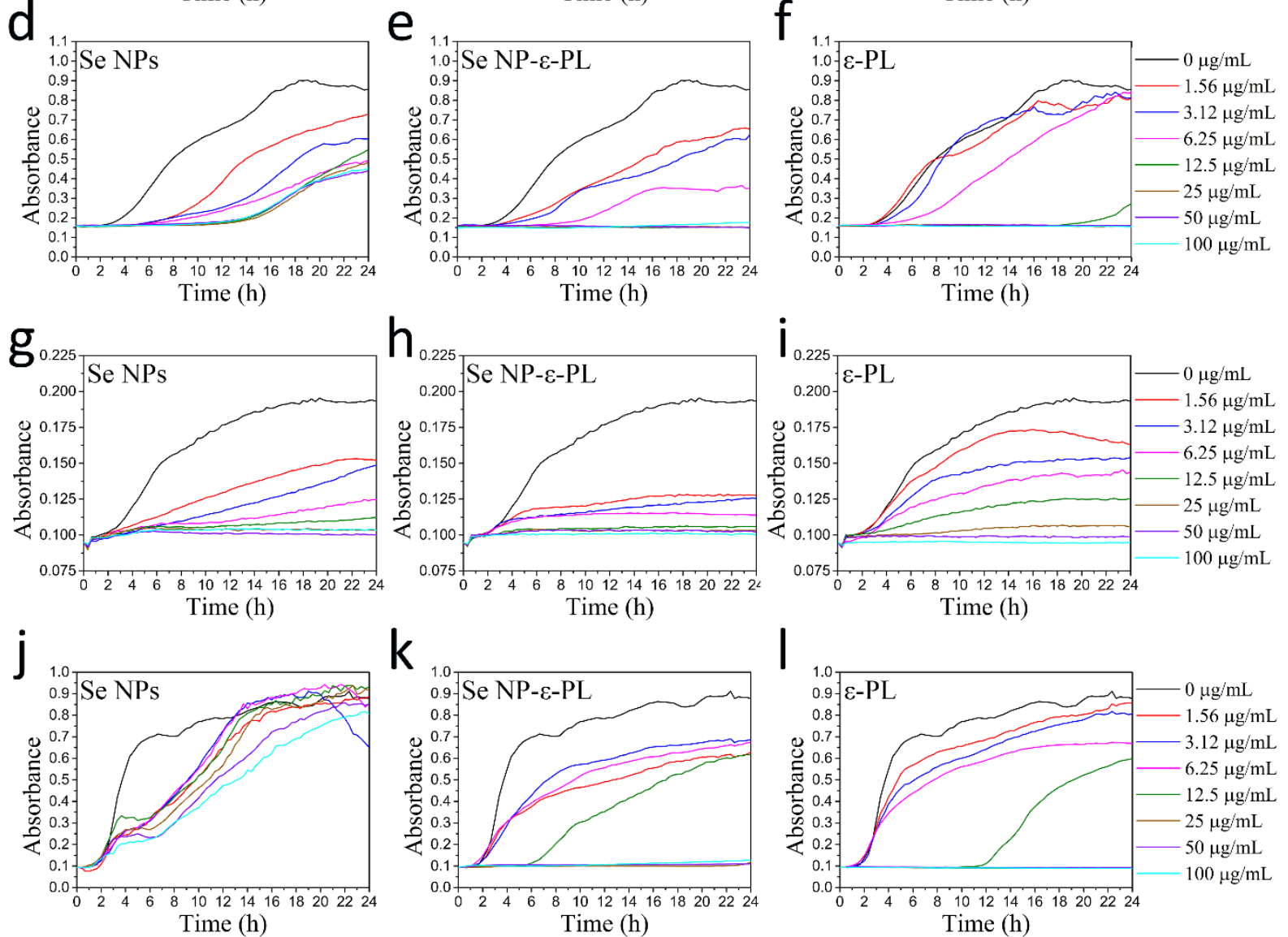

Figure S1. Growth curves of bacteria in MHB with different weight concentrations of Se NPs,

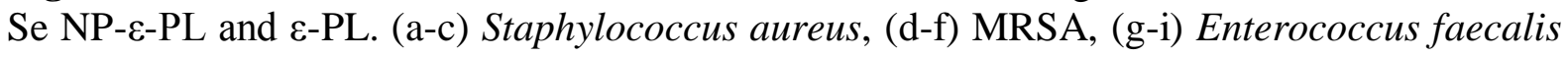
and (j-1) Escherichia coli. 

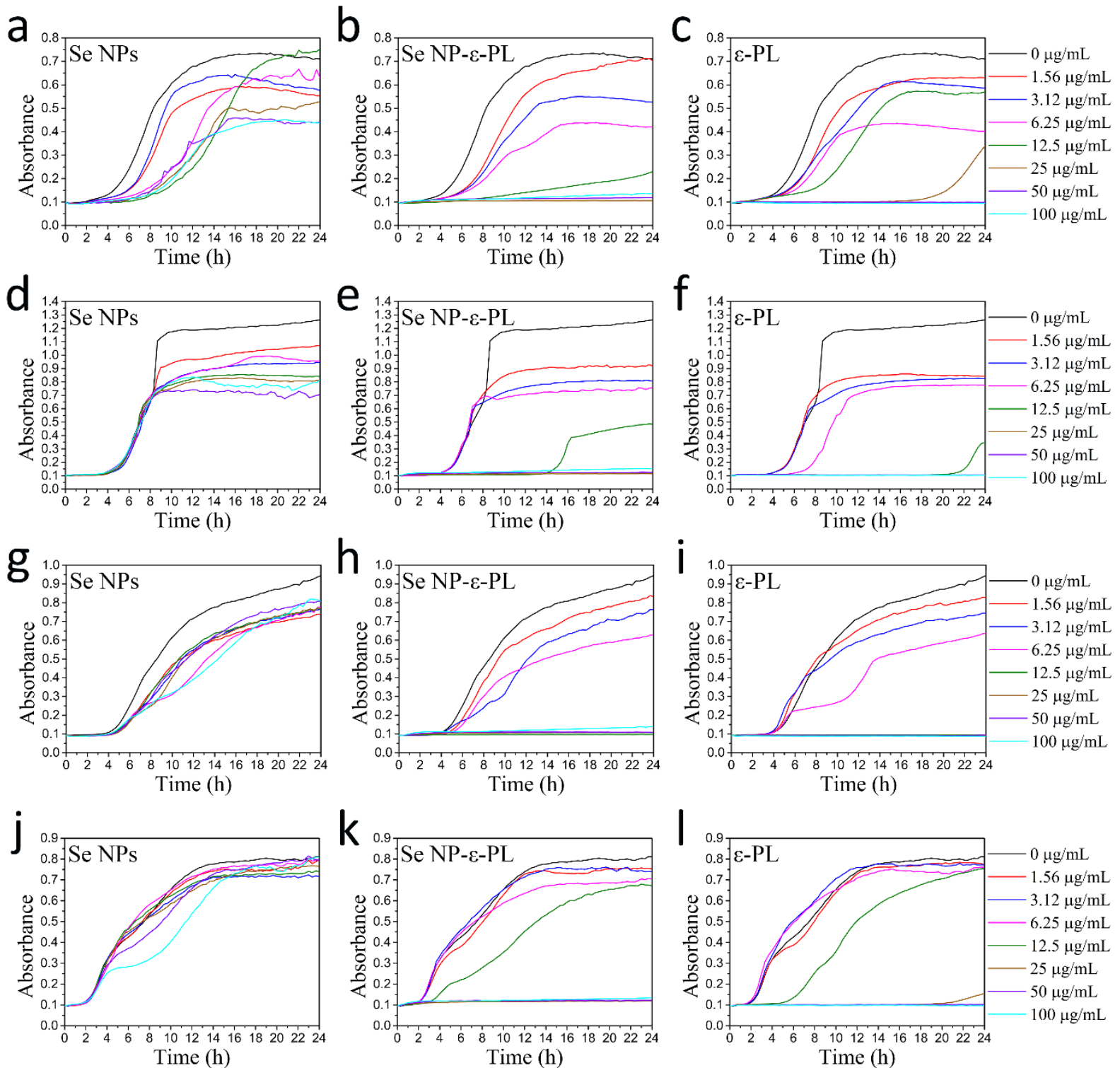

Figure S2. Growth curves of bacteria in MHB with different weight concentrations of Se NPs,

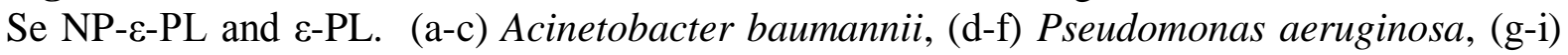
Klebsiella pneumoniae and (j-1) Klebsiella pneumoniae (MDR). 

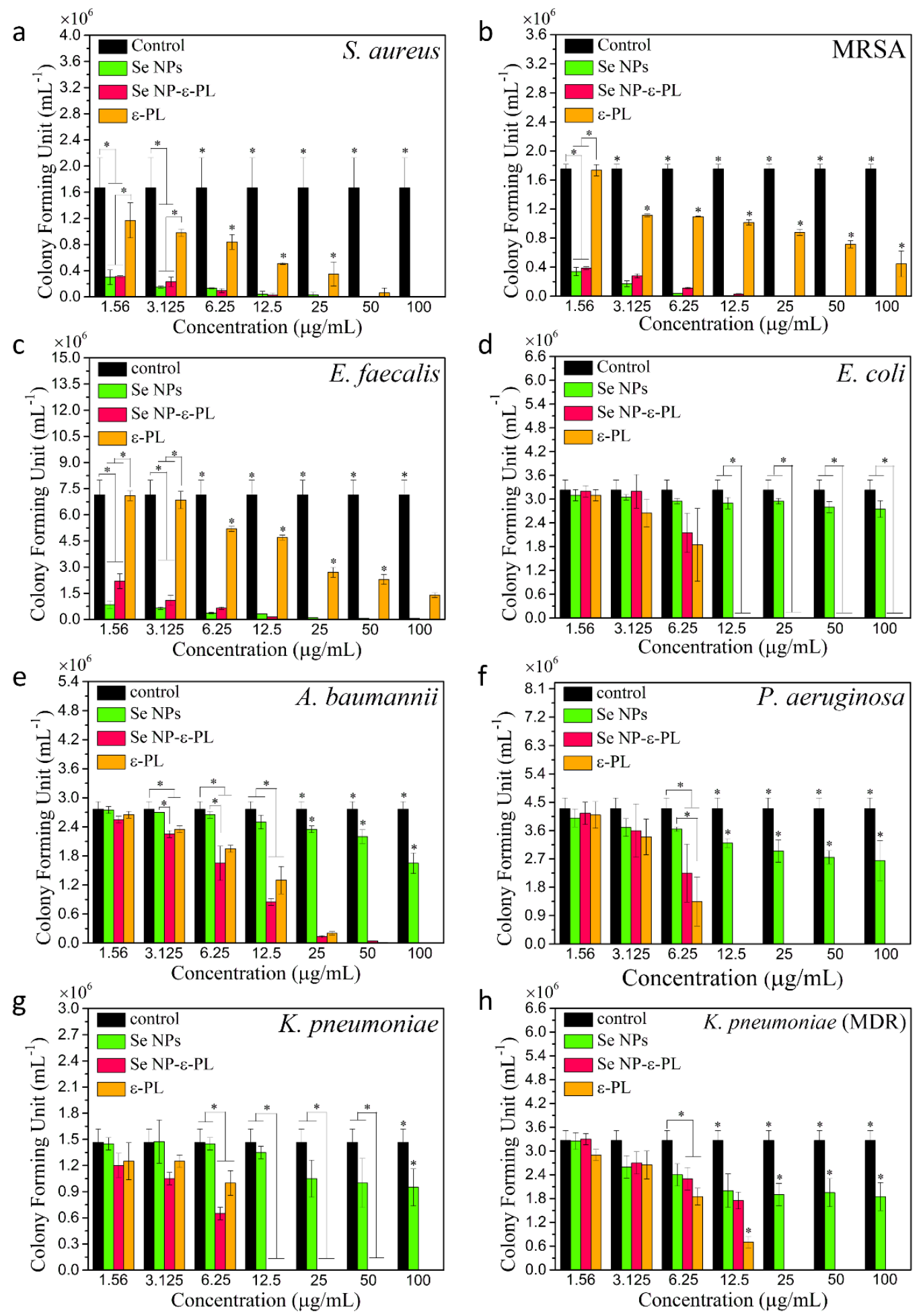

Figure S3. Colony forming unit (CFU) assay results for eight different strains of bacteria. (a) Staphylococcus aureus, (b) MRSA, (c) Enterococcus faecalis, (d) Escherichia coli, (e) Acinetobacter baumannii, (f) Pseudomonas aeruginosa, (g) Klebsiella pneumoniae and (h) Klebsiella pneumoniae (MDR) in MHB with no treatment (control) or with different concentrations of Se NPs, Se NP- $\varepsilon$-PL or pure $\varepsilon$-PL. One-way ANOVA analysis was adopted to compare means of experimental groups, * represents $\mathrm{p}$-value $<0.05$. The asterisk(s) directly marked on a bar indicate(s) this group is significantly different to all other groups at the same concentration. 

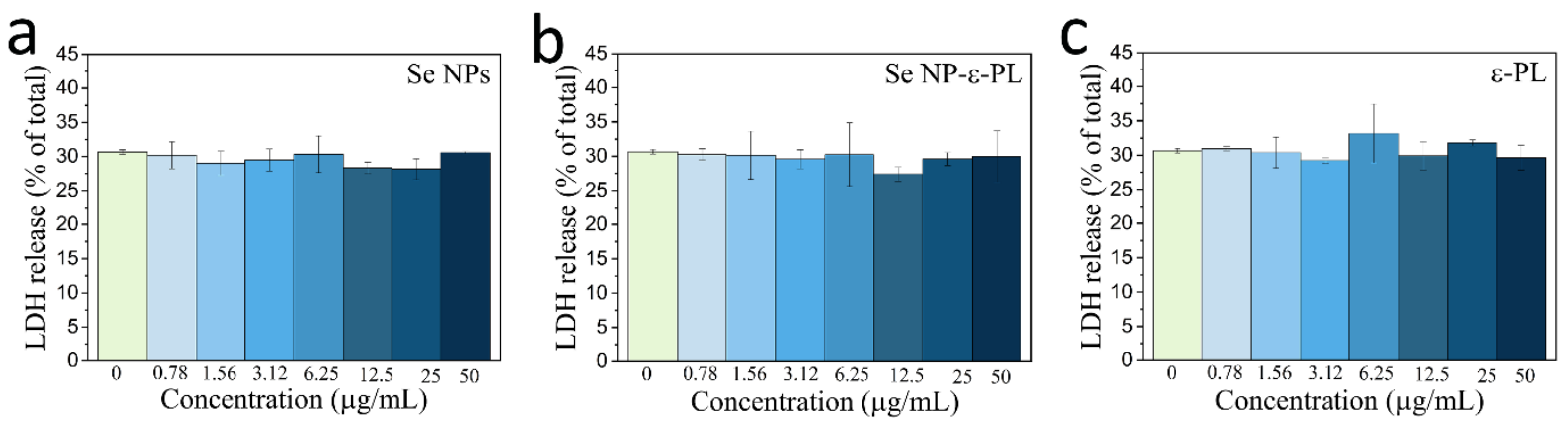

Figure S4. LDH test results on HDFs after $6 \mathrm{~h}$ exposure to different concentrations of testing materials. (a) Se NPs, (b) Se NP- $\varepsilon$-PL and (c) $\varepsilon-\mathrm{PL}$. LDH is an enzyme present within the mitochondria of living cells, and as such increased levels of the LDH in the culture medium imply rupture of the cell membranes. Cells treated with Se NPs, Se NP- $\varepsilon$-PL or $\varepsilon$-PL for $6 \mathrm{~h}$ showed no significant differences to the untreated control, indicating these treatments at concentrations up to $50 \mu \mathrm{g} / \mathrm{mL}$ do not cause obvious damage to the cell membranes of the HDFs over the $6 \mathrm{~h}$ period.
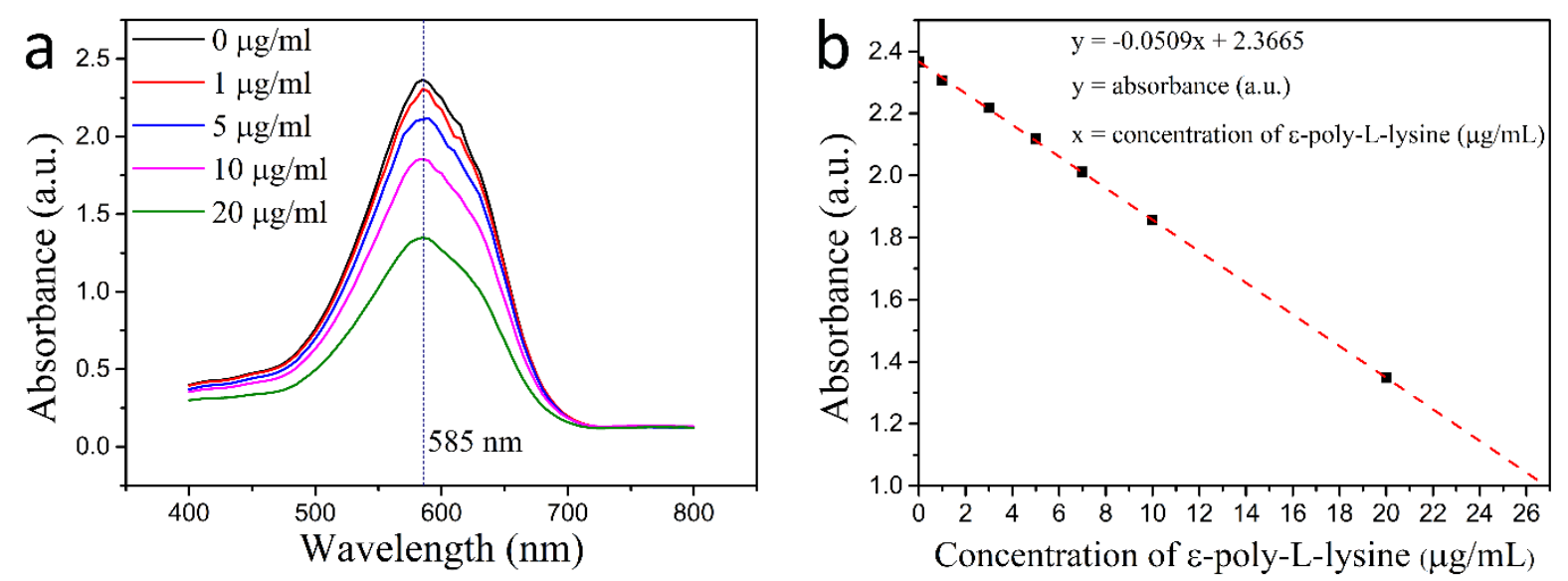

Figure S5. Methods for measuring the concentration of $\varepsilon$-PL. (a) the UV spectra of the supernatant obtained from $\varepsilon$-PL and trypan blue mixed solution after $1 \mathrm{~h}$ incubation at $37{ }^{\circ} \mathrm{C}$, (b) the standard curve of $\varepsilon$-PL concentration. 

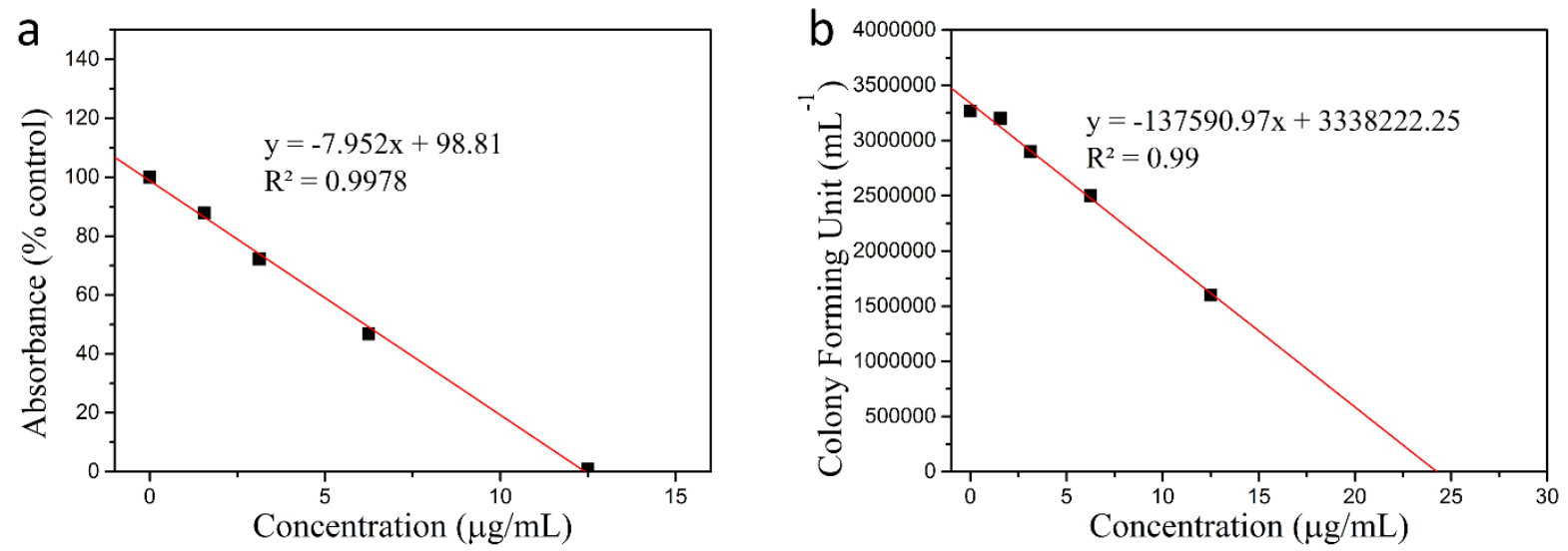

Figure S6. Method for MIC and MBC calculation. (a) Method for MIC determination. Sample concentration-absorbance (\% control) and linear regression analysis used to determine MIC values. MIC was determined as the concentration at which absorbance (\% control) becomes zero. The example shown was based on experimental data obtained for Se NP- $\varepsilon$-PL against $K$. Pneumoniae in MHB. The calculated MIC value was $12.43 \mu \mathrm{g} / \mathrm{mL}$. The average values were taken from two independent tests. (b) Method for MBC determination. Sample concentrationkilling curves and linear regression analysis used to determine MBC values. MBC was determined as the concentration at which colony-forming unit $(\mathrm{CFU}) / \mathrm{mL}$ becomes zero. The example shown was based on experimental data obtained for Se NP- $\varepsilon$-PL against $K$. Pneumoniae (MDR) in MHB. The calculated MBC value was $24.26 \mu \mathrm{g} / \mathrm{mL}$. The average values were taken from two independent tests.

\section{Supporting table}

Table S1. Zeta potentials of different bacteria

\begin{tabular}{cccccc}
\hline Bacteria type & S. aureus & E. faecalis & E. coli & A. baumannii & K. pneumoniae \\
\hline $\begin{array}{c}\text { Mean zeta } \\
\text { potential }\end{array}$ & $-8.9 \pm 2.6$ & $-10.2 \pm 0.7$ & $-19.2 \pm 0.4$ & $-9.1 \pm 0.2$ & $-21.4 \pm 0.8$ \\
$(\mathrm{mV})$ & & & &
\end{tabular}

* All data are expressed as mean \pm standard deviation (s.d.) of three measurements.

\section{References}

(1) ISO. 10993-5: Biological evaluation of medical devices. Tests for in vitro cytotoxicity 2009. 